\title{
How many r-process components? A view from the mirror image of s-process studies
}

\author{
R. Gallino ${ }^{1}$, G.J. Wasserburg ${ }^{2}$, M. Busso ${ }^{3}$, and O. Straniero ${ }^{4}$ \\ ${ }^{1}$ Dipartimento di Fisica Generale, Via P. Giuria 1, Torino 10125, Italy \\ email: gallino@ph.unito.it, \\ ${ }^{2}$ California Institute of Technology Pasadena (CA, USA) \\ email: gjw@gps.caltech.edu, \\ ${ }^{3}$ Dip. Fisica, Universitá di Perugia (Italy) \\ email: maurizio.busso@fisica.unipg.it, \\ ${ }^{4}$ Teramo Observatory, INAF, Teramo 64100, Italy \\ email: straniero@oa-teramo.inaf.it,
}

\begin{abstract}
The classical analysis of the s-process is commonly used to predict the s percentage contribution to the solar system abundance of a given isotope, and by default of the r-process residual (calculated as $1 .-\mathrm{s}$ ). We discuss the advantages and the disadvantages of this first-order prediction, by comparing stellar model calculations at various metallicities in AGB stars and in massive stars exploding as SNII, with spectroscopic observations of different stellar populations. Observations of short-lived r-process isotopes in the early solar system help to characterize at least three different r-process components.
\end{abstract}

Keywords. Nuclear reactions, nucleosynthesis, abundances, stars: abundances, stars: AGB, stars: Population II

\section{A plurality of s-process components}

Since the pioneering work of Burbidge et al. (1957), heavy isotopes beyond iron that are built up by neutron captures have been divided according to their slow (s-process) or rapid ( $r$-process) time scales, as compared to those of $\beta$-decays from unstable nuclei encountered along the neutron capture path. For the s-process, steady flow conditions are prevailing far from magic nuclei (Clayton 1968), implying for unbranched isotopes the condition $\sigma(A-1) N_{s}(A-1) \approx \sigma(A) N_{s}(A)$, where $\langle\sigma(A) v>$ indicates the Maxwellian averaged product of cross section and relative velocity. The curve $\sigma(A) N_{s}(A)$ appears generally smooth, but interrupted by steep decreases in correspondence of magic neutron numbers $N=50,82$ or 128 , where the neutron capture cross sections are very small and the resulting s-process abundances are large. This happens at $\mathrm{Sr}, \mathrm{Y}, \mathrm{Zr}$, at $\mathrm{Ba}$ to $\mathrm{Nd}$ and at the termination of the s-process involving $\mathrm{Pb}$ and $\mathrm{Bi}$. Three s-process components were anticipated by the classical analysis (Clayton and Ward 1974; Käppeler et al. 1982): the weak, the main, and the strong s-component.

The main s-component is the outcome of many generations of asymptotic giant Branch stars (AGB) polluting the interstellar medium before the solar system formed. Actually, the main s-component is far from being a unique process! Fig. 1 shows AGB model predictions for an initial mass of $1.5 M_{\odot}$ as a function of metallicity $[\mathrm{Fe} / \mathrm{H}]$ and for different ${ }^{13} \mathrm{C}$ pocket efficiencies (Busso et al. 1999; Gallino et al. 2005 and references therein). The predicted light-s $[\mathrm{ls} / \mathrm{Fe}]$ indicator with $\mathrm{ls}=(\mathrm{Y}, \mathrm{Zr})$ and the predicted heavy$\mathrm{s}$ indicator $[\mathrm{hs} / \mathrm{Fe}]$ with $\mathrm{hs}=(\mathrm{Ba}, \mathrm{La}, \mathrm{Nd}, \mathrm{Sm})$ are shown in the upper panels, while the 

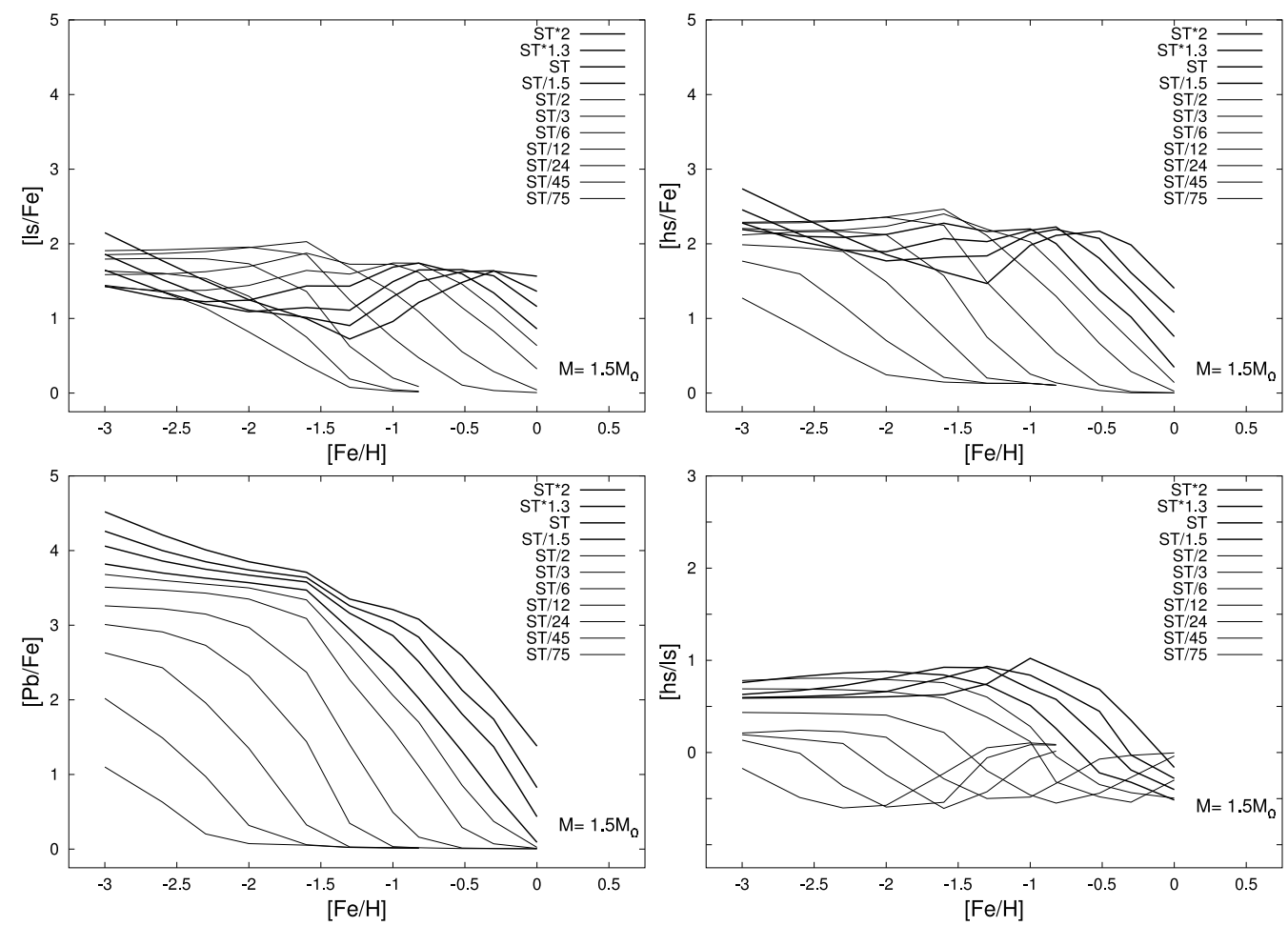

Figure 1. AGB model predictions for an initial mass of $1.5 M_{\odot}$ and different ${ }^{13} \mathrm{C}$ pocket efficiencies: $[\mathrm{ls} / \mathrm{Fe}]$ versus $[\mathrm{Fe} / \mathrm{H}]$ (top-left panel); $[\mathrm{hs} / \mathrm{Fe}]$ versus $[\mathrm{Fe} / \mathrm{H}]$ (top-right panel); $[\mathrm{Pb} / \mathrm{Fe}]$ versus $[\mathrm{Fe} / \mathrm{H}]$ (bottom-left panel); [hs/ls] versus $[\mathrm{Fe} / \mathrm{H}]$ (bottom-right panel).

predicted third s-indicator $[\mathrm{Pb} / \mathrm{Fe}]$ at the termination of the s-process path is shown in the bottom-left panel. The intrinsic indicator [hs/ls] is shown in the bottom-right panel. The two intrinsic indexes $[\mathrm{hs} / \mathrm{ls}]$, and $[\mathrm{Pb} / \mathrm{hs}]$, are independent of the fact that the observed s-enhanced star is an intrinsic AGB or an extrinsic AGB, this last resulting by mass transfer in a close binary system.

The major neutron source in AGB stars is provided by the primary-like ${ }^{13} \mathrm{C}(\alpha, \mathrm{n}){ }^{16} \mathrm{O}$ neutron source occurring in the He-rich zone of AGB stars in radiative conditions during the interpulse phase. The main difficulty is that of finding some mixing mechanism to inject a small amount of protons from the envelope in the top layers of the He-rich and ${ }^{12} \mathrm{C}$-rich intershell region, where, at $\mathrm{H}$ re-ignition, they would be captured by the abundant ${ }^{12} \mathrm{C}$, producing ${ }^{13} \mathrm{C}$ (see Goriely, these Proceedings). As a matter of fact, in most calculations of recent years the extent of the proton-enriched layers and/or the ${ }^{13} \mathrm{C}$ concentration in them have been assumed as free parameters, to be calibrated by observational constraints. A way to obtain the formation of a ${ }^{13} \mathrm{C}$ pocket was first elaborated by Herwig (2000), introducing an exponential diffusive overshoot at the borders between the convective and the radiative zones. A different algorithm based on a velocity profile at the inner border of the convective envelope during the development of a third dredge-up episode has been developed by Straniero et al. (2005; see also Cristallo et al., these Proceedings). Also in this case the formation of a ${ }^{13} \mathrm{C}$ pocket results, and a more extensive work in this direction is highly desirable. A second minor neutron source, by the reaction ${ }^{22} \mathrm{Ne}(\alpha, \mathrm{n})^{25} \mathrm{Mg}$, operates during the recurrent thermal instabilities in the He shell, when the bottom temperature in the convective shell reaches values of $\sim 3 \times 10^{8} \mathrm{~K}$. The ${ }^{22} \mathrm{Ne}$ neutron source governs the final yields in correspondence of s-process branchings. 


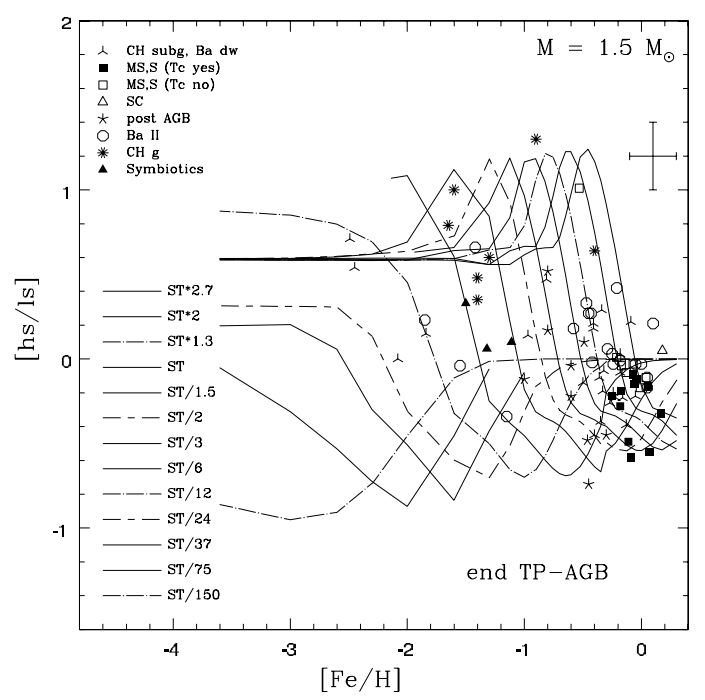

Figure 2. Comparison of AGB model predictions of $[\mathrm{hs} / \mathrm{ls}]$ versus $[\mathrm{Fe} / \mathrm{H}]$ for different ${ }^{13} \mathrm{C}$ pocket efficiencies and spectroscopic observations of different intrinsic and extrinsic AGB stars.

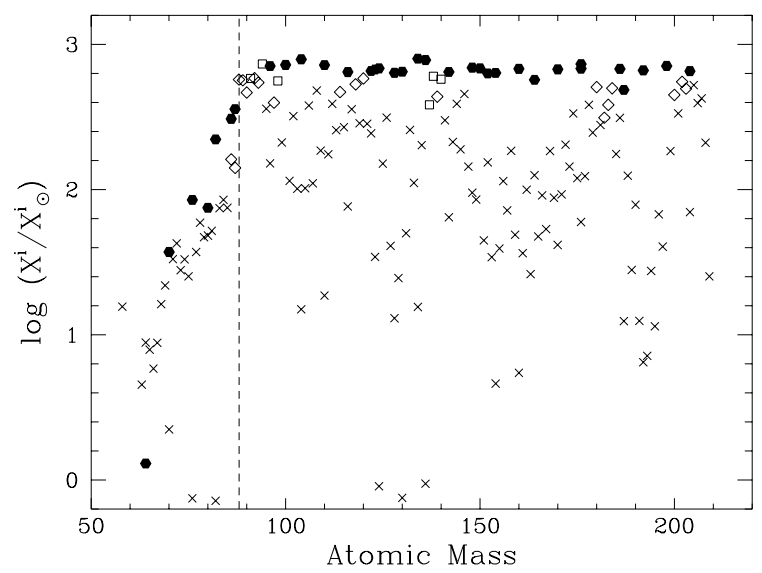

Figure 3. Reproduction of the main component by an AGB model of half solar metallicity and ST case for the ${ }^{13} \mathrm{C}$ pocket efficiency (from Gallino et al. 1988).

In Fig. 1, for a given metallicity the concentration of the ${ }^{13} \mathrm{C}$ source has been left to vary over a wide interval, as indicated by the labels. Here ST refers to the abundance of ${ }^{13} \mathrm{C}$ assumed as standard by Gallino et al. (1998). Different ${ }^{13} \mathrm{C}$-pocket choices are conceivably caused by different stellar physical conditions like rotation, initial mass, mass loss rate. Note that only the results of case ST were reported in the Fig. 12 of Busso et al. (1999) when describing the trend versus $[\mathrm{Fe} / \mathrm{H}]$ of the elemental abundances of the three major s-peaks. For a given ${ }^{13} \mathrm{C}$-pocket choice (e.g., case ST of Fig. 1) at a solar metallicity the $1^{\text {st }}$ s-process peaks at $\mathrm{Sr}-\mathrm{Y}-\mathrm{Zr}$. As shown in Fig. 3, for a half-solar metallicity a best-fit to the main s-component in the solar system is obtained, which includes all the s-only isotopes between $A \sim 90$ and 204 (Gallino et al. 1998; Arlandini et al. 1999). For any metallicity, a best-fit to the main s-component can be found for the same $[\mathrm{hs} / \mathrm{ls}]=-0.2$ value that is obtained for the case ST at half-solar metallicity, provided a suitable ${ }^{13} \mathrm{C}$-pocket strength is adopted. Comparison of AGB stellar model 


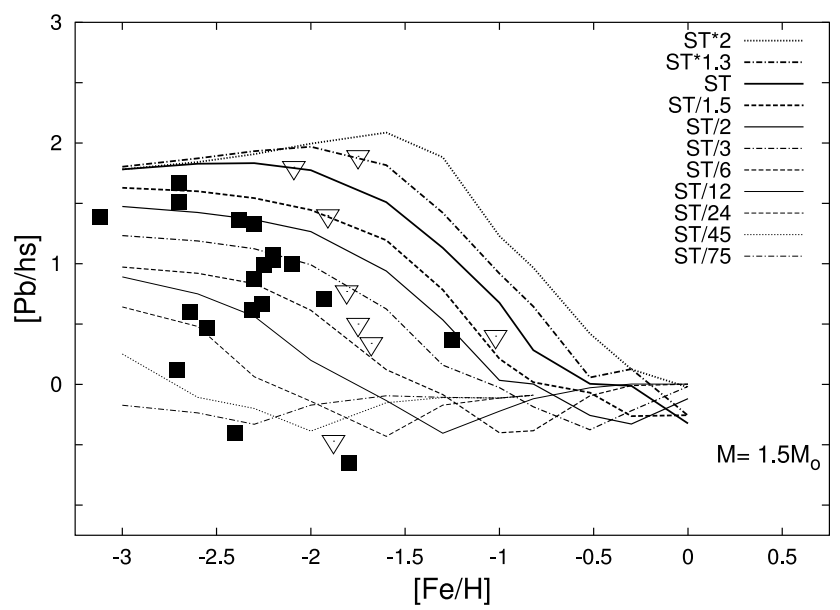

Figure 4. Comparison of AGB model predictions of $[\mathrm{Pb} / \mathrm{hs}]$ versus $[\mathrm{Fe} / \mathrm{H}]$ for different ${ }^{13} \mathrm{C}$ pocket efficiencies and recent spectroscopic observations of metal-poor Lead stars. Full squares are from the literature cited by Delaude et al.(2004), Barbuy et al. 2005; Ivans et al. (2005); open triangles are from Lucatello (2003, and These proceedings).

calculations at various metallicities with spectroscopic observations of different stellar populations of both intrinsic AGBs and extrinsic AGBs is shown in Fig. 2 for the [hs/ls] intrinsic indicator versus metallicity. It clearly demonstrates the vaste multiplicity of s-process components (Busso et al. 2001).

\subsection{The strong s-component and the low-metallicity Lead stars}

By further decreasing the metallicity, the neutron captures accumulate at the termination point of the s-process, feeding first the s-peak at $\mathrm{Ba}$ to $\mathrm{Sm}$, and then the $3^{r d}$ s-peak at $\mathrm{Pb}-\mathrm{Bi}$. A major evidence of a spread of ${ }^{13} \mathrm{C}$-pocket efficiencies is provided by the low-metallicity C-rich and s-process rich stars (Lead stars). This is apparent in Fig. 4 where the intrinsix index $[\mathrm{Pb} / \mathrm{hs}]$ is shown as a function of $[\mathrm{Fe} / \mathrm{H}]$. As a matter of fact, below $[\mathrm{Fe} / \mathrm{H}]=-2$, a large spread of $[\mathrm{Pb} / \mathrm{hs}]$, by two orders of magnitudes, is predicted, and this in nice agreement with spectroscopic observations. In conclusion, the strong scomponent in the solar system is the result of all previous generations of halo AGB stars, which mostly produce $\mathrm{Pb}$ (essentially all solar ${ }^{208} \mathrm{~Pb}$ ) and $20 \%$ of solar Bi (Travaglio et al. 2001).

Among the couple of dozen of very metal-poor stars C-rich and s-process rich discovered so far there is a consistent number of objects showing the unusual characteristic of being also r-process rich. Example are HE2148-1247 (Cohen et al. 2003) and CS29497-030 (Ivans et al. 2005). Vanhala and Cameron (1998) showed through numerical simulations how the supernova ejecta may interact with a nearby molecular cloud, polluting it with fresh nucleosynthesized material and triggering the condensation of binary system of low mass. Note that according to Lucatello et al. (2005) all C-rich and s-rich metal-poor stars show binarity from their radial velocity temporal variations. The subsequent evolution of such close binary systems, and how the pre-enrichment of r-process elements influences the s-process occurring in the more massive AGB companion, is discussed by Ivans et al. (2005, also these Proceedings) and by Bisterzo et al. (these Proceedings). 


\subsection{The weak s-component}

Massive stars during their hydrostatic convective core He burning phase and especially during the subsequent convective shell C-burning phase are the sites of the weak scomponent (Rauscher et al. 2002; Woosley et al. 2002). ${ }^{22} \mathrm{Ne}$ is the major neutron source during core He burning. The ${ }^{22} \mathrm{Ne}$ abundance derives from the transmutation of all CNO original nuclei into ${ }^{14} \mathrm{~N}$ during previous $\mathrm{H}$ burning followed by double $\alpha$ capture on ${ }^{14} \mathrm{~N}$ in the early phases of He burning. During convective shell $\mathrm{C}$ burning the major neutron source is primary ${ }^{13} \mathrm{C}(\alpha, n){ }^{16} \mathrm{O}$, with ${ }^{13} \mathrm{C}$ deriving from proton captures of ${ }^{12} \mathrm{C}$, and protons and $\alpha$ particles being released by the major channels of C-burning (Pignatari et al., these Proceedings). The major neutron poison during C-burning is primary ${ }^{16} \mathrm{O}$. This means the the weak s-component decreases linearly with decreasing the metallicity. Some primary production of light neutron capture isotopes below $A \approx 90$ is the consequence of explosive nucleosynthesis. In particular, the average constant [ $\mathrm{Sr}, \mathrm{Y}, \mathrm{Zr} / \mathrm{Fe}]$ versus $[\mathrm{Fe} / \mathrm{H}]$ shown by halo stars requires a consistent primary contribution to the solar abundance of these elements, by the order of 20-30\%, originated in massive stars (Travaglio et al. 2004). Further studies are needed to investigate more carefully this problem.

\subsection{Presolar grains of $A G B$ origin}

The analysis of presolar $\mathrm{SiC}$ and corundum grains of AGB origin recovered from pristine carbonaceous meteorites (e.g., Lugaro et al. 2003; Zinner and Amari 1999; Lodders and Amari 2005; Wasserburg et al. 2005, and references therein) demonstrates once more that a plurality of AGB stars, each one having suffered a different neutron exposure, are at the origin of these meteoritic presolar grains, which condense in the outflows of AGB mass-losing envelopes.

\section{Short-lived isotopes in the Early Solar System and a multiplicity of r-process components}

Experimental evidence of short-lived r-process isotopes in the early solar system help to characterize at least three different r-process components. The two short-lived isotopes: ${ }^{129} \mathrm{I}(\bar{\tau}=23 \mathrm{Myr})$ and ${ }^{182} \mathrm{Hf}(\bar{\tau}=13 \mathrm{Myr})$, which are most of r-process origin, have been shown to be alive in the early solar system with a ratio incompatible with a unique r-process distribution (Wasserburg et al. 1996). A third r-process component from meteoritic studies derives from a further conflict existing from a refined analysis of the actinides ${ }^{244} \mathrm{Pu}(\bar{\tau}=115 \mathrm{Myr})$ and new upper limits on ${ }^{247} \mathrm{Cm}(\bar{\tau}=22.5 \mathrm{Myr})$ in the early solar system with respect to the heavy r-process ${ }^{182} \mathrm{Hf}$ (Wasserburg et al. 2005).

\section{Spectroscopic discoveries of r-process enhanced very metal-poor stars}

Spectroscopic observations of ultra metal-poor and r-process enriched stars agree with the independent meteoritic studies on short-lived nuclei. The r-process distribution shown in the heavy r-process elements beyond Ba by a few extremely r-process enhanced stars is in strict agreement with the r-process residuals in the solar system as deduced from the studies of the concurrent s-process. However, in these stars, the light elements below Ba, like Ag, $\mathrm{Pd}, \mathrm{Cd}$, show abundances below the above r-process expectations (Cowan et al. 2005 and references therein; T. Beers, these Proceedings). Moreover, recent spectroscopic analyses of r-process-rich ultra metal-poor stars of the ratio $\mathrm{Th} / \mathrm{Eu}$, or $\mathrm{Pb} / \mathrm{Eu}$, point out the necessity of a breakdown of the idea of a unique heavy r-process distribution beyond Ba (Hill et al. 2002; François et al. 2003; Plez et al. 2004). 


\section{Conclusion}

This quick analysis shows that neutron capture process like the classical s-process and the r-process show in Nature a plurality of components, both from observations of high-resolution spectroscopy of very-metal poor stars, from the analysis of meteoritic presolar grains, and from studies of short-lived isotopes detected in early solar system condensates.

\section{References}

Arlandini, C. et al. 1999, ApJ 525, 886

Barbuy, B. et al. 2005, A\&BA 429, 1031

Burbidge, E.M., Burbidge, G.R., Fowler, W.A., \& Hoyle, F., 1957 Rev. Mod. Phys. 29, 547

Busso, M., Gallino, R., Wasserburg, G.J. 1999, Annu. Rev. Astron. Astrophys. 37, 239

Busso, M., Lambert, D.L., Gallino, R., Travaglio, C., Smith V.V, 2001, ApJ 557, 802

Busso, M., Straniero, O., Gallino, R., Abia, C. 2004, in Origin and Evolution of the Elements, eds. A. McWilliam and M. Rauch, Carnegie Observatories Astrophysics Series 4, 67

Clayton, D.D. 1968, Principles of Stellar Evolution and Nucleosynthesis (Chicago: University of Chicago Press), pp 612

Clayton, D.D., Ward, R.A. 1974, ApJ 193, 397

Cohen, J.G., Christlieb, N., Qian, Y.Z., Wasserburg, G.J. 2003, ApJ 588, 1082

Cowan, J.J. et al. 2005, ApJ 627, 238

Delaude, D. et al. 2004, Mem. Soc. Astron. It. 75, 706

François, P. et al. 2003, A\&3A 403, 1105

Gallino, R. et al. 1998, ApJ 497, 388

Gallino, R., Delaude, D., Husti, L., Cristallo, S., Straniero, O., Ryan, S. 2005, Nuclear Phys. A, in press

Herwig, F. 2000, $A \mathscr{E} A, 360,952$

Hill, V. et al. 2002, A\&A 387, 560

Ivans, I.I., Sneden, C., Gallino, R., Cowan, J.J., Preston, G.W. 2005, ApJ 627, L145

Johnson, J.A., Bolte, M. 2004, ApJ 605, 462

Käppeler, F. et al. 1982, ApJ 257, 821

Lodders, K., amari, S. 2005, Chemie der Erde 65, 93

Lucatello, S. 2003, PhD Thesis, University of Padova

Lucatello, S. et al. 2003, AJ 125, 875

Lucatello, S., Tsangarides, S., Beers, T.C., Carretta, E., Gratton, R., Ryan, S.G. 2005, ApJ 625, 825

Lugaro, M. et al. 2003, ApJ 593, 1056

Plez, B. et al. 2004, A\& $A$ 428, L9

Rauscher, T., Heger, A., Hoffman, R.D., Woosley, S.E. 2002, ApJ 576, 323

Straniero, O., Gallino, R., Cristallo, S. 2005, Nuclear Phys. A, in press

Travaglio, C., Gallino, R., Busso, M., Gratton, R. 2001, ApJ 549, 346

Travaglio, C., Gallino, R., Cowan, J.J., Jordan, F., Sneden, C. 2004, ApJ 601, 864

Vanhala, H.A.T. and Cameron, A.G.W. 1998, ApJ 508, 291

Wasserburg, G.J., Busso, M., Gallino, R. 1996, ApJ 466, L109

Wasserburg, G.J., Busso, M., Gallino, R., Nollett, K.M. 2005, Nucl. Phys. A in press

Woosley, S.E., Heger, A., Weaver, T.A 2002, Rev. Mod. Phys. 74, 1015

Zinner, E., Amari, S. 1999 in Asymptotic Giant Branch Stars, IAU Symp. 191, ed. T. Le Bertre, A. Lèbre, C. Waelkens, 59 